Applied Mathematics and Mechanics (English Edition), 2006, 27(3):417-424

(C)Editorial Committee of Appl. Math. Mech., ISSN 0253-4827

\title{
DAMPING OF VERTICALLY EXCITED SURFACE WAVE IN WEAKLY VISCOUS FLUID *
}

JIAN Yong-jun (营永军 $)^{1,2}, \quad$ E Xue-quan (鄂学全) ${ }^{2}, \quad$ ZHANG Jie (张杰) $)^{1,3}$

( 1. First Institute of Oceanography, State Oceanic Administration, Qingdao 266061, Shandong Province, P. R. China;

2. Institute of Mechanics, Chinese Academy of Sciences, Beijing 100080, P. R. China;

3. Key Laboratory of Marine Science and Numerical Modeling, State Ocean Administration,

Qingdao 266061, Shandong Province, P. R. China)

(Communicated by DAI Shi-qiang)

\begin{abstract}
In a vertically oscillating circular cylindrical container, singular perturbation theory of two-time scale expansions is developed in weakly viscous fluids to investigate the motion of single free surface standing wave by linearizing the Navier-Stokes equation. The fluid field is divided into an outer potential flow region and an inner boundary layer region. The solutions of both two regions are obtained and a linear amplitude equation incorporating damping term and external excitation is derived. The condition to appear stable surface wave is obtained and the critical curve is determined. In addition, an analytical expression of damping coefficient is determined. Finally, the dispersion relation, which has been derived from the inviscid fluid approximation, is modified by adding linear damping. It is found that the modified results are reasonably closer to experimental results than former theory. Result shows that when forcing frequency is low, the viscosity of the fluid is prominent for the mode selection. However, when forcing frequency is high, the surface tension of the fluid is prominent.
\end{abstract}

Key words: vertically forced oscillation; viscous damping; weakly viscous fluid

Chinese Library Classification: 0353.2

2000 Mathematics Subject Classification: 76B15; 76B07; 76M45

Digital Object Identifier(DOI): 10.1007/s 10483-006-0319-1

\section{Introduction}

E Xuequan et al. ${ }^{[1-3]}$ carried out the flow visualization and experimental study on surface wave patterns in a circular cylindrical vessel by vertical external vibrations. They obtained very beautiful photographs of free surface patterns. Recently, a theoretical analysis associated with the experiments of Refs.[1-3] was carried out by Jian Yongjun et al..$^{[4-7]}$, from which a nonlinear amplitude equation, the second order free surface displacements and their contours of the surface waves were obtained by two-time scale singular perturbation expansion in ideal fluids. Due to ignoring viscid dissipation in their theoretical analysis, the forced frequency had large discrepancies between theoretical and experimental results. Due to ignoring viscid dissipation in their theoretical analysis, the forced frequency had large discrepancies between theoretical and experimental results. Hill ${ }^{[8]}$ studied the Faraday resonance of interfacial waves in a two-layer, weakly viscous system in a rectangular domain, and gave analytical expression of damping coefficient. This paper will consider the influence of weak viscosity on the pattern

* Received Jun.9, 2004; Revised Oct.16, 2005

Project supported by the National Natural Science Foundation of China (Nos.19772063, 19772068) and the Doctoral Research Fund of the Ministry of Education (No.20010141024)

Corresponding author JIAN Yong-jun, Doctor, E-mail: jianyongjun@yahoo.com.cn 
formation. The purpose is to determine damping coefficient analytically in a vertical oscillating circular cylinder, which is often obtained empirically. We divide whole stream fields into outer potential flow region and inner boundary layer region. Both solutions in these two domains are derived by two-time scale expansion. The damping coefficient is deduced analytically by solvability condition of higher order solution. By modifying the dispersive relation obtained from Ref.[6], the theoretical forced frequency is much closer to experimental result than that of without taking weak viscosity into account for the same flow pattern both in theory and experiment.

\section{Governing Equation and Boundary Conditions in Viscous Fluid}

We still consider surface waves excited by the vertical motion of a circular cylindrical basin filled with weak viscous fluid. The choice of the coordinate system is the same as that in Ref.[4]. Supposing the fluid is incompressible, we have

$$
\nabla \cdot v=0
$$

where $v=v(r, \theta, z, t)$ is the velocity field of fluid and obeys the Navier-Stokes equation,

$$
\frac{\partial v}{\partial t}+(\boldsymbol{v} \cdot \nabla) v=-\frac{1}{\rho} \cdot \nabla \pi+\nu \nabla^{2} v
$$

where $\pi(r, \theta, z, t)=p(r, \theta, z, t)-p_{0}+\rho\left(g-\ddot{z}_{0}\right) z, \nu$ is the kinematic viscosity and other parameters are the same as that in Ref.[4]. At the free surface $z=\eta(r, \theta, t)$, ignoring the effect of surface tension, the kinematic condition is

$$
\left.w(r, \theta, z, t)\right|_{z=\eta}=\frac{\partial \eta}{\partial t}+\boldsymbol{v} \cdot \nabla \eta
$$

Normal part perpendicular to the free surface and the tangential part of the free surface are

$$
\begin{aligned}
& \left.\pi(r, \theta, z, t)\right|_{z=\eta}-\rho g\left(g-\ddot{z}_{0}\right) \eta-2 \rho \nu \frac{\partial w}{\partial z}=0 \\
& \rho \nu\left(\frac{\partial u}{\partial z}+\frac{\partial w}{\partial r}\right)=\rho \nu\left(\frac{1}{r} \cdot \frac{\partial w}{\partial \theta}+\frac{\partial v}{\partial z}\right)=0
\end{aligned}
$$

where $u$ and $v$ denote the velocity components in $r$-and $\theta$-direction respectively. At the bottom and on the side-wall of the container, one must use the no-slip condition,

$$
v(r, \theta, z, t)=0 .
$$

Under the condition of linear inviscid fluid and irrotational motion, we have $\pi(r, \theta, z, t)=-\frac{\partial \phi}{\partial t}$, where $\phi(r, \theta, z, t)$ is inviscid velocity potential. As for the linear viscous problem, it is reasonable to separate the velocity into potential flow and boundary layer velocity (velocity vector $U$ is relevant to boundary layers),

$$
v=\nabla \phi+U
$$

Substituting $\pi(r, \theta, z, t)$ and Eq.(7) into Eq.(2), and ignoring the nonlinear terms, we obtain following diffusive equation with respect to the velocity of boundary layer,

$$
\frac{\partial U}{\partial t}=\nu \nabla^{2} \boldsymbol{U}
$$

Take Eq.(7) into Eq.(1), boundary layer velocity satisfies zero divergence condition,

$$
\nabla \cdot \boldsymbol{U}=0
$$


Substituting Eq.(7) into Eq.(1) and Eqs.(3)-(6), and neglecting all nonlinear terms, governing equations and boundary conditions can be expressed as

$$
\begin{aligned}
& \nabla^{2} \phi=0, \quad-h \leq z \leq \eta(r, \theta, t), \quad 0<r<R, \\
& \frac{\partial \eta}{\partial t}-\frac{\partial \phi}{\partial z}=W, \quad z=\eta, \\
& \frac{\partial \phi}{\partial t}+g\left(1+\frac{4 A \omega_{0}^{2}}{g} \cdot \cos 2 \omega_{0} t\right) \eta+2 \nu\left(\frac{\partial^{2} \phi}{\partial z^{2}}+\frac{\partial W}{\partial z}\right)=0, \quad z=\eta, \\
& \rho \nu\left(\frac{\partial u}{\partial z}+\frac{\partial w}{\partial r}\right)=\rho \nu\left(\frac{1}{r} \cdot \frac{\partial w}{\partial \theta}+\frac{\partial v}{\partial z}\right)=0, \quad z=\eta, \\
& \nabla \phi+U=0, \quad z=-h \text { and } r=R,
\end{aligned}
$$

where $\nabla^{2}$ is three-dimensional Laplace operator in cylindrical coordinate, $W$ is the component of boundary layer velocity $U$ in $z$-direction.

\section{Non-dimensionalized Equations and Curve Coordinates}

Taking the radius $R$ of the tank as the length scale, non-dimensionalizing all related independent variables and unknown variables, the following scalings are adopted:

$$
\begin{aligned}
& z^{*}=z / R, r^{*}=r / R, \eta^{*}=\eta / R, t^{*}=t / \sqrt{R / g}, \phi^{*}=\phi /(R \sqrt{g R}), A^{*}=A / R, \\
& \omega_{0}^{*}=\omega_{0} / \sqrt{g / R}, \varepsilon^{*}=4 A \omega_{0}^{2} / g, \nu^{*}=\nu /\left(\varepsilon^{* 2} R \sqrt{g R}\right), U^{*}=U / \sqrt{g R} .
\end{aligned}
$$

The above asterisks denote dimensionless quantities and that the asterisks are subsequently dropped. The parameter $\varepsilon^{*}$ quantifies the acceleration due to the vertical oscillation relative to gravity and is assumed to be much less than unity. In order to facilitate the theoretical analysis, we supposed that the viscosity is weak, and has been scaled as $\varepsilon^{2}$ in Eq.(15). Substituting Eq.(15) into Eqs.(8)-(14), the following dimensionless governing equations and boundary conditions are obtained:

$$
\begin{aligned}
& \nabla^{2} \phi=0, \quad-h / R \leq z \leq \eta(r, \theta, t), \quad 0<r<1, \\
& \frac{\partial \eta}{\partial t}-\frac{\partial \phi}{\partial z}=W, \quad z=\eta \\
& \left\{\begin{array}{c}
\varepsilon^{2}\left(2 \frac{\partial^{2} \phi}{\partial r \partial z}+\frac{\partial W}{\partial r}\right)+\varepsilon^{2} \frac{\partial U}{\partial z}=0, \\
\varepsilon^{2}\left(\frac{2}{r} \cdot \frac{\partial^{2} \phi}{\partial \theta \partial z}+\frac{1}{r} \cdot \frac{\partial W}{\partial \theta}\right)+\varepsilon^{2} \frac{\partial V}{\partial z}=0, \quad z=\eta
\end{array}\right. \\
& \frac{\partial \phi}{\partial t}+\left(1+\epsilon \cos 2 \omega_{0} t\right) \eta+2 \nu \varepsilon^{2}\left(\frac{\partial^{2} \phi}{\partial z^{2}}+\frac{\partial W}{\partial z}\right)=0, \quad z=\eta \\
& \nabla \phi+U=0, \quad z=-h / R \text { and } r=1 .
\end{aligned}
$$

In addition, boundary layer equations become

$$
\begin{aligned}
& \frac{\partial \boldsymbol{U}}{\partial t}=\varepsilon^{2} \nu \nabla^{2} \boldsymbol{U}, \\
& \nabla \cdot \boldsymbol{U}=0,
\end{aligned}
$$

where $U$ and $V$ are boundary layer velocity components in $r$ - and $\theta$-directions, respectively.

Next we establish a boundary layer coordinate system. Recalling that the order of viscosity 


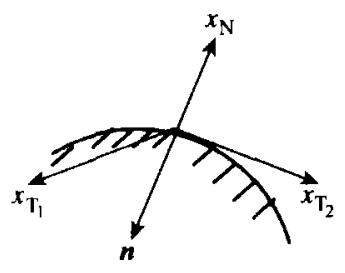

Fig.1 Curve coordinates of the boundary layer

$\nu$ is $O\left(\varepsilon^{2}\right)$, and the thickness of an osciallatory boundary layer has the order $O\left\{(2 \nu / \Omega)^{1 / 2}\right\}$, so the order of the boundary layer thickness is $O(\varepsilon)$. A boundary layer coordinate is introduced as shown in Fig.1, where $x_{\mathrm{N}}$ is the normal direction pointing into the fluid from the wall, hence, opposite to $n . x_{\mathrm{T}_{1}}$ and $x_{\mathrm{T}_{2}}$ determined tangential plane that is perpendicular to the normal direction $x_{\mathrm{N}}$. So $x_{\mathrm{N}}, x_{\mathrm{T}_{1}}$ and $x_{\mathrm{T}_{2}}$ form a locally rectangular coordinate system. We introduce a new boundary layer variable $\varsigma=x_{N} / \varepsilon$, then the continuity equation (22) in terms of the local coordinate becomes

$$
-\frac{\partial \boldsymbol{n}}{\partial \varsigma} \cdot \boldsymbol{U}+\varepsilon\left(\frac{\partial U_{\mathrm{T}_{1}}}{\partial x_{\mathrm{T}_{1}}}+\frac{\partial U_{\mathrm{T}_{2}}}{\partial x_{\mathrm{T}_{2}}}\right)=0
$$

In the case of weak viscosity supposition, boundary layer equation (21) becomes

$$
\frac{\partial \boldsymbol{U}}{\partial t}=\nu \frac{\partial^{2} \boldsymbol{U}}{\partial \varsigma^{2}}
$$

\section{$3 \quad O\left(\varepsilon^{0}\right)$ Approximate Solutions}

\subsection{External potential flow solution of $O\left(\varepsilon^{0}\right)$ equations}

We invoke two-time scale expansions, a slowly varying time scale $\tau$ is introduced, such that $\tau=t \varepsilon$. Expanding velocity potential $\phi$, free surface displacement $\eta$ and boundary layer velocity $U$ into power series of the small parameter $\varepsilon$, and expanding Eqs.(17) and (19) into Taylor series about $z=0$, if we neglect higher-order terms $O\left(\varepsilon^{2}\right)$, and compare the power of small parameter $\varepsilon^{0}$ at the two hands of Eqs.(16)-(20), we obtain the following $O\left(\varepsilon^{0}\right)$ potential flow governing equation and boundary conditions:

$$
\begin{aligned}
& \nabla^{2} \phi_{0}=0, \quad-h / R \leq z \leq \eta(r, \theta, t), \quad 0<r<1 \\
& \frac{\partial \eta_{0}}{\partial t}-\frac{\partial \phi_{0}}{\partial z}=0, \quad z=0 \\
& \frac{\partial \phi_{0}}{\partial t}+\eta_{0}=0, \quad z=0 \\
& \frac{\partial \phi_{0}}{\partial r}=0, \quad r=1 \\
& \frac{\partial \phi_{0}}{\partial z}=0, \quad z=-h / R .
\end{aligned}
$$

Boundary condition (26) ignores the normal velocity $W$ of the boundary layer because it will work in the meniscus region (this region is defined as the overlap between free surface and the side wall boundary layer) near the wall, and will be modified in solving $O\left(\varepsilon^{1}\right)$ potential flow. Boundary conditions (28) and (29) overlook the normal velocity of the boundary layer on the side-wall and at the bottom. By separation of variables, the $O\left(\varepsilon^{0}\right)$ velocity potential and free surface displacement can be expressed as

$$
\begin{aligned}
& \phi_{0}=\mathrm{J}_{m}(\lambda r) \cosh [\lambda(z+h / R)] \cdot\left[p(\tau) \mathrm{e}^{\mathrm{i} \Omega t}+\bar{p}(\tau) \mathrm{e}^{-\mathrm{i} \Omega t}\right] \cos m \theta, \\
& \eta_{0}=-\mathrm{i} \Omega \mathbf{J}_{m}(\lambda r) \cosh \lambda \cdot\left[p(\tau) \mathrm{e}^{\mathrm{i} \Omega t}-\bar{p}(\tau) \mathrm{e}^{-\mathrm{i} \Omega t}\right] \cos m \theta,
\end{aligned}
$$

here $\bar{p}(\tau)$ denotes the complex conjugate of $p(\tau)$, "i" is the unit of an imaginary number. $\mathrm{J}_{m}(r)$ is the $m$-order Bessel function of the first kind. The wave number $\lambda=\lambda_{m n}$ satisfies $d \mathbf{J}_{m}\left(\lambda_{m n} r\right) /$ $\left.d r\right|_{r=1}=0$ and is the $n$th positive roots. Substituting Eqs.(30) and (31) to Eq.(26), the following dispersive relation should be obeyed:

$$
\Omega^{2}=\lambda_{m n} \tanh \left(\lambda_{m n} h / R\right)=\lambda \tanh (\lambda h / R) .
$$




\subsection{Internal boundary layer solution of $O\left(\varepsilon^{0}\right)$ equations}

Boundary layer regions are composed of side-wall boundary layer and bottom boundary layer, and every boundary layer has three velocity components. Let $U_{0 \mathrm{~W}}, V_{0 \mathrm{~W}}$ and $W_{0 \mathrm{~W}}$ become the three velocity components of side-wall boundary layer, we know the velocity $U_{0 \mathrm{~W}}=0$ from Eqs.(20) and (28). The appropriate fast coordinate is introduced by $\varsigma_{\mathrm{W}}=(r-1) / \varepsilon$, the velocity component $V_{0 \mathrm{~W}}$ in the direction of $\theta$ satisfies the following modified equation and corresponding boundary conditions:

$$
\begin{aligned}
& \frac{\partial V_{0 \mathrm{~W}}}{\partial t}=\nu \frac{\partial^{2} V_{0 \mathrm{~W}}}{\partial \varsigma_{\mathrm{W}}^{2}}, \\
& V_{\mathrm{OW}}=-\left.\frac{1}{r} \cdot \frac{\partial \phi_{0}}{\partial \theta}\right|_{r=1}, \quad \varsigma_{\mathrm{W}}=0, \\
& V_{\mathrm{OW}} \rightarrow 0, \quad \varsigma_{\mathrm{W}} \rightarrow \infty .
\end{aligned}
$$

The method of separation of variables was utilized again. Substituting Eq.(30) into Eq.(34), the solution of Eqs.(33)-(35) can be formulated as follows:

$$
\begin{aligned}
V_{0 \mathrm{~W}}= & m \mathrm{~J}_{m}(\lambda) \cosh [\lambda(z+h / R)] \sin m \theta \\
& \cdot\left[p(\tau) \mathrm{e}^{\mathrm{i} \Omega t} \mathrm{e}^{-(1+\mathrm{i}) \sqrt{\frac{\Omega}{2 \nu}} \varsigma \mathrm{w}}+\bar{p}(\tau) \mathrm{e}^{-\mathrm{i} \Omega t} \mathrm{e}^{-(1-\mathrm{i}) \sqrt{\frac{\Omega}{2 \nu}} \varsigma \mathrm{w}}\right] .
\end{aligned}
$$

In the same way, the solution $W_{0 \mathrm{~W}}$ of $z$-direction on the side-wall can be written as

$$
\begin{aligned}
& W_{0 \mathrm{~W}}=-\lambda \mathrm{J}_{m}(\lambda) \sinh [\lambda(z+h / R)] \cos m \theta \\
& \cdot\left[p(\tau) \mathrm{e}^{\mathrm{i} \Omega t} \mathrm{e}^{-(1+\mathrm{i}) \sqrt{\frac{\Omega}{2 \nu}} \varsigma \mathrm{w}}+\bar{p}(\tau) \mathrm{e}^{-\mathrm{i} \Omega t} \mathrm{e}^{-(1-\mathrm{i}) \sqrt{\frac{\Omega}{2 \nu}} \varsigma \mathrm{w}}\right] .
\end{aligned}
$$

Likewise, by the coordinate transformation $\varsigma_{\mathrm{B}}=(z+h / R) / \varepsilon$, three velocity components $U_{0 \mathrm{~B}}$, $V_{0 \mathrm{~B}}$ and $W_{0 \mathrm{~B}}$ of bottom boundary layer can be obtained. we know the velocity $W_{0 \mathrm{~B}}=0$ from Eqs. (20) and (28), and $U_{0 \mathrm{~B}}$ and $V_{0 \mathrm{~B}}$ are

$$
\begin{aligned}
U_{0 \mathrm{~B}}= & -\left(\frac{m}{r} \mathrm{~J}_{m}(\lambda r)-\lambda \mathrm{J}_{m+1}(\lambda r)\right) \cos m \theta \\
& \cdot\left[p(\tau) \mathrm{e}^{\mathrm{i} \Omega t} \mathrm{e}^{-(1+\mathrm{i}) \sqrt{\frac{\Omega}{2 \nu}} \varsigma_{\mathrm{B}}}+\bar{p}(\tau) \mathrm{e}^{-\mathrm{i} \Omega t} \mathrm{e}^{-(1-\mathrm{i}) \sqrt{\frac{\Omega}{2 \nu}} \varsigma_{\mathrm{B}}}\right], \\
V_{0 \mathrm{~B}}= & \frac{m}{r} \mathrm{~J}_{m}(\lambda r) \sin m \theta \cdot\left[p(\tau) \mathrm{e}^{\mathrm{i} \Omega t} \mathrm{e}^{-(1+\mathrm{i}) \sqrt{\frac{\Omega}{2 \nu}} \varsigma_{\mathrm{B}}}+\bar{p}(\tau) \mathrm{e}^{-\mathrm{i} \Omega t} \mathrm{e}^{-(1-\mathrm{i}) \sqrt{\frac{\Omega}{2 \nu}} \varsigma_{\mathrm{B}}}\right] .
\end{aligned}
$$

\section{$4 O\left(\varepsilon^{1}\right)$ Equation, Amplitude Equation and Damping Coefficient}

Comparing the power of small parameter $\varepsilon^{1}$ at the two hands of Eqs.(16)-(20), we obtain following $O\left(\varepsilon^{1}\right)$ governing equation and boundary conditions:

$$
\begin{aligned}
& \nabla^{2} \phi_{1}=0, \quad-h / R<z<0, \quad 0<r<1, \\
& \frac{\partial \eta_{1}}{\partial t}-\frac{\partial \phi_{1}}{\partial z}=-\frac{\partial \eta_{0}}{\partial \tau}+\frac{\left.W_{0 \mathrm{~W}}\right|_{z=0}}{\varepsilon}, \quad z=0, \\
& \frac{\partial \phi_{1}}{\partial t}+\eta_{1}=-\frac{\partial \phi_{0}}{\partial \tau}-\cos \left(2 \omega_{0} t\right) \eta_{0}, \quad z=0, \\
& \frac{\partial \phi_{1}}{\partial r}=-U_{1 \mathrm{~W}}=-\left.\int_{\infty}^{\varsigma_{\mathrm{W}}}\left(\frac{1}{r} \cdot \frac{\partial V_{0 \mathrm{~W}}}{\partial \theta}+\frac{\partial W_{0 \mathrm{~W}}}{\partial z}\right)\right|_{r=1} d \varsigma_{\mathrm{W}}, \quad \varsigma_{\mathrm{W}}=0, \\
& \frac{\partial \phi_{1}}{\partial z}=-W_{1 \mathrm{~B}}=-\left.\int_{\infty}^{\varsigma_{\mathrm{B}}}\left(\frac{\partial U_{0 \mathrm{~B}}}{\partial r}+\frac{U_{0 \mathrm{~B}}}{r}+\frac{1}{r} \frac{\partial V_{0 \mathrm{~B}}}{\partial \theta}\right)\right|_{z=-h / R} d \varsigma_{\mathrm{B}}, \quad \varsigma_{\mathrm{B}}=0 .
\end{aligned}
$$


Since the $O\left(\varepsilon^{1}\right)$ problem is inhomogeneous and the $O\left(\varepsilon^{0}\right)$ homogeneous problem had a nontrivial solution, in order to solve $O\left(\varepsilon^{1}\right)$, a so-called solvability condition must be satisfied. The detailed approach is that Eq.(40) multiplied by the complex conjugate of $\phi_{0}$ and subtract $\phi_{1}$ multiplied by the complex conjugate of Eq.(25), then integrate this equation in the region filled fluid. The volume integral can be transformed into surface integral by using Green's theorem, and finally we derive the following amplitude equation $p(\tau)$ associated with slowly varying time $\tau$ :

$$
\frac{d p(\tau)}{d \tau}=-\mathrm{i} \alpha \bar{p}(\tau)+\beta p(\tau)
$$

here $\alpha$ is a positive real number, and it represents the influence of vertically external excitation; $\beta$ denotes the complex-valued damping coefficient. The detailed expressions of $\alpha$ and $\beta$ are

$$
\begin{aligned}
& \alpha=\frac{\Omega}{4}, \beta=\beta_{\mathrm{W}}+\beta_{\mathrm{B}}+\beta_{\mathrm{M}}, \quad \beta_{\mathrm{W}}=\frac{\lambda\left[\sinh \frac{2 \lambda h}{R}+\frac{2 \lambda h}{R}\right]}{8 \Omega \cosh ^{2} \frac{\lambda h}{R}} \sqrt{\frac{2 \nu}{\Omega}}(1+\mathrm{i}), \\
& \beta_{\mathrm{B}}=\frac{\lambda^{2}}{\left[4 \Omega \cosh ^{2} \frac{\lambda h}{R}\right]} \sqrt{\frac{2 \nu}{\Omega}}(1+\mathrm{i}), \quad \beta_{\mathrm{M}}=\frac{\lambda^{2} \Omega}{\left[2\left(\lambda^{2}-m^{2}\right)\right]} \cdot \sqrt{\frac{2 \nu}{\Omega}}(1+\mathrm{i}),
\end{aligned}
$$

where $\beta_{\mathrm{W}}, \beta_{\mathrm{B}}$ and $\beta_{\mathrm{M}}$ represent the viscous damping in the side-wall, the bottom boundary layer, and the meniscus region, respectively. Henderson ${ }^{[9]}$ pointed out that the real and the imaginary part of damping in Eq.(46) mean the value of the damping and frequency shift, respectively. The damping of the real part causes the attenuation of the surface wave, while the imaginary part changes the natural frequency of the surface wave.

\section{Critical Condition to Appear Stable Surface Wave}

Separating Eq.(45) into real and imaginary parts and letting $p(\tau)=p_{1}(\tau)+\mathrm{i} p_{2}(\tau), \beta=$ $\beta_{1}+\mathrm{i} \beta_{2}$, then Eq.(45) can be expressed as

$$
\begin{aligned}
& d p_{1}(\tau) / d \tau=\beta_{1} p_{1}(\tau)-\left(\alpha+\beta_{2}\right) p_{2}(\tau), \\
& d p_{2}(\tau) / d \tau=-\left(\alpha-\beta_{2}\right) p_{1}(\tau)+\beta_{1} p_{2}(\tau)
\end{aligned}
$$

Ordinary differential equation groups (47) and (48) are linear and their instability condition can be easily obtained as

$$
\alpha^{2}>|\beta|^{2},
$$

where $|\beta|$ represents the absolute value of $\beta$. The physical meaning of Eq.(49) is that the vertically external excitation can not be smaller than the damping of the fluid if the surface wave occurs. The natural frequency, with the surface tension being considered in Ref.[6], is modified by introducing the imaginary part of the viscous damping coefficient $\beta_{2}\left(\beta_{2}\right.$ is the damping sum of side-wall, bottom and meniscus), thus we have

$$
\hat{\Omega}=\sqrt{\lambda \tanh (\lambda h / R)\left(1+\Gamma / \rho \cdot \lambda^{2}\right)}-\beta_{2} .
$$

\section{Computational Results}

\subsection{Determination of critical curve}

We illustrate a critical curve with respect to dimensional forced frequency and forced amplitude in Fig.2 by considering the dispersive relation (32) and instable condition (49). The unstable region is above the curve in Fig.2. It shows from Fig.2 that when the excited frequency is small, the excited amplitude must be large enough to produce a surface wave. However, when the forced frequency is high, the forced amplitude must be small. If both forced frequency and amplitude are high, the parameter $\varepsilon$ cannot be less than unity, and our present theoretical assumption cannot be satisfied. 


\subsection{Influence of viscous damping and surface tension on mode selection}

In order to present the influence of surface tension and damping on the pattern selection, we gave a curve of frequency via wave number in Fig.3. It can be seen from Fig.3 that when the

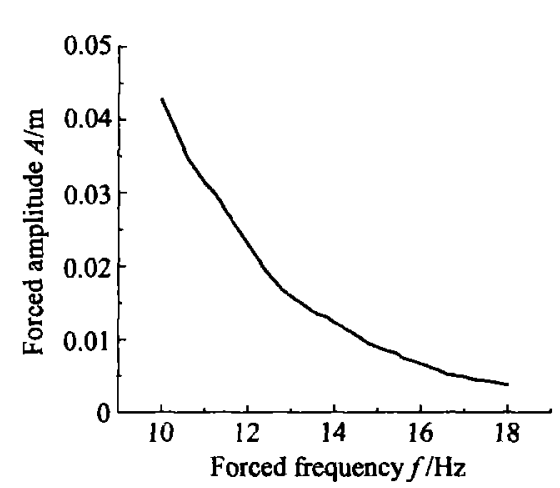

Fig.2 Critical curve associated with excited amplitude and forced frequency $(h=1.0 \mathrm{~cm}, R=7.5 \mathrm{~cm}, \nu=$ $\left.10^{-6} \mathrm{~m}^{2} / \mathrm{s}\right)$

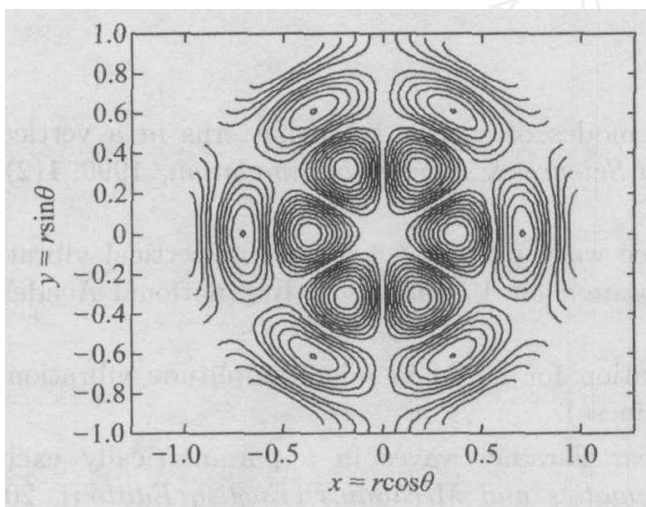

(a) $(3,3)$ mode, (left) forced frequency $f=19.9 \mathrm{~Hz}$, (right) forced frequency $f=20 \mathrm{~Hz}$

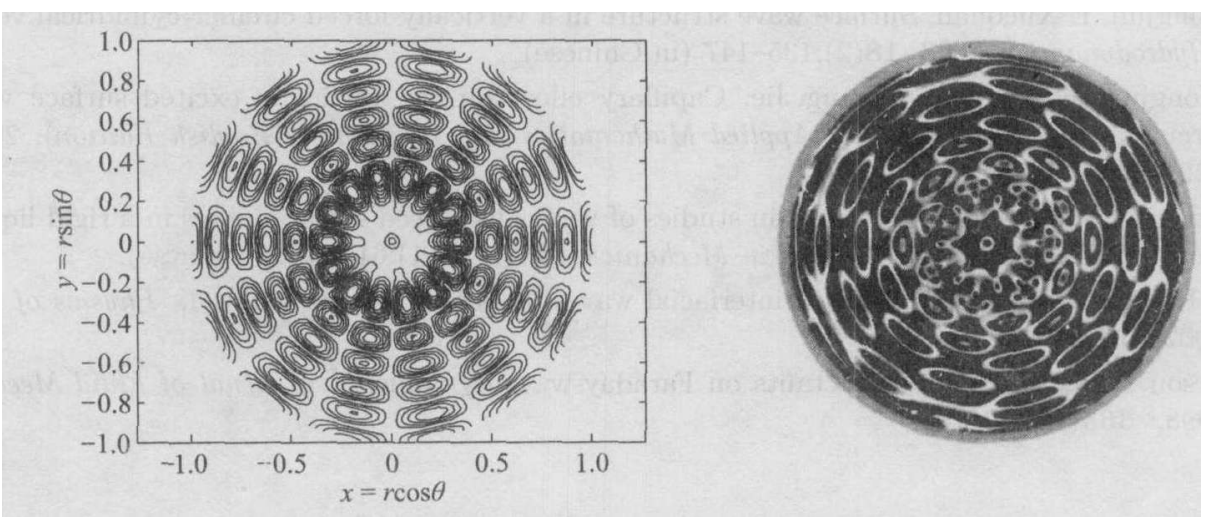

(b) $(7,6)$ mode, (left) forced frequency $f=26.7 \mathrm{~Hz}$, (right) forced frequency $f=52 \mathrm{~Hz}$

Fig.4 Comparison of theoretical contours of surface wave mode with those of experiment $\left(h=1.0 \mathrm{~cm}, R=7.5 \mathrm{~cm}, A=11.4 \mu \mathrm{m}, \nu=10^{-6} \mathrm{~m}^{2} / \mathrm{s}, \Gamma=0.0727 \mathrm{~N} / \mathrm{m}, \rho=10^{3} \mathrm{~kg} / \mathrm{m}^{3}\right.$ ) 
forcing frequency is low, the viscosity of the fluid is prominent for the mode selection. However, when the forcing frequency is high, the surface tension of the fluid is prominent.

\subsection{Comparison with experiment}

Figure 4 illustrates the comparison of the theoretical contours including the effect of surface tension and viscous damping in different forced frequency with experimental contours. It is found from Fig.4 that when the wave number is low, e.g. $(3,3)$ mode, the forced frequency agrees well with the experimental result. However, when the wave number is high, e.g. $(7,6)$ mode, the forced frequency had large discrepancies. The reason is possibly due to the influence of contact line and mode competition, which are ignored in our present analysis.

\section{Conclusions}

From above analysis, following results can be obtained:

(i) The method of two-time scale expansion is effective to resolve the problem with the effect of weak viscosity in vertically oscillating circular cylindrical container.

(ii) The analytical expression of the damping coefficient and the critical condition to appear stable surface wave are obtained.

(iii) When the wave number is small, the influence of the viscous damping on pattern selection is insignificant. However, when the wave number is large, the effect of the surface tension is important.

(iv) It is found that the modified results including weak viscosity and surface tension are reasonably closer to experimental results than former theory without considering their effects.

\section{References}

[1] E Xuequan, Gao Yuxin. Ordered and chaotic modes of surface wave patterns in a vertically oscillating fluid[J]. Communications in Nonlinear Sciences \& Numerical Simulation, 1996, 1(2):16.

[2] E Xuequan, Gao Yuxin. Visualization of surface wave patterns of a fluid in vertical vibration [C]. In: Proceedings of the Fourth Asian Symposium on Visualization. International Academic Publishers, Beijing, 1996, 653-658.

[3] Gao Yuxin, E Xuequan. Surface wave visualization for liquid in micro-amplitude vibration[J]. Experimental Mech, 1998, 13(3):326-333 (in Chinese).

[4] Jian Yongjun, E Xuequan, Bai Wei. Nonlinear Faraday waves in a pararnetrically excited circular cylindrical container[J]. Applied Mathematics and Mechanics (English Edition), 2003, 24(10):1194-1207.

[5] Jian Yongjun, E Xuequan. Surface wave structure in a vertically forced circular cylindrical vessel [J]. J Hydrodynamics, 2003, 18(2):135-147 (in Chinese).

[6] Jian Yongjun, E Xuequan, Zhang Jie. Capillary effect on the vertically excited surface wave in a circular cylindrical vessel[J]. Applied Mathematics and Mechanics (English Edition), 2006, 27(2):227-234.

[7) Jian Yongjun, E Xuequan. Advances in studies of vertically forced surface waves in a rigid liquidfilled container: a review[J]. Progress in Mechanics, 2004, 34(1):61-76 (in Chinese).

[8] Hill D F. The Faraday resonance of interfacial waves in weakly viscous fluids[J]. Physics of Fluids, 2002, 14(1):158-169.

[9] Henderson D M. Effects of surfactants on Faraday-wave dynamics[J]. Journal of Fluid Mechanics, $1998, \mathbf{3 6 5}(12): 89-107$. 